\title{
Impact of Oculomotor Retraining on the Visual Perception of Curvature
}

\author{
Joel Miller and Leon Festinger \\ Program in Visual Perception \\ New School for Social Research
}

\begin{abstract}
Observers viewed a computer-generated display consisting of horizontally oriented, concave-up curved lines. The position of these curves was contingent on the horizontal position of the eye so that, in order to change fixation errorlessly, from one point to another on the curve, the eye would have to execute a purely horizontal movement. In Condition $\mathrm{H}$ this was achieved by moving the curves horizontally, so that the minimum point was always at the horizontal eye position location, thus simulating the effect of viewing a line through a wedge prism on a contact lens. In Condition V it was achieved by moving the curves vertically so that the point fixated always had the same vertical location. In both conditions eye movements were reprogrammed rapidly to eliminate the vertical components of the saccades that were present at the start. While a small, but significant, amount of perceptual adaptation was obtained in Condition $\mathrm{H}$, none at all was obtained in Condition $\mathrm{V}$. The results are interpreted as not in support of such theories of perceptual adaptation to curvature distortion as require a close relationship between motor learning and perceptual change.
\end{abstract}

There has been a long-standing interest in the problem of how visual perception alters with prolonged exposure to optically rearranged retinal stimulation. The importance of the question lies in its implications for theories concerning the development and the plasticity of the visual perceptual system.

The early landmark studies, reported by Stratton (1896, 1897), concerned his experiences wearing a monocular (one eye occluded) optical device that inverted the retinal image. Many interpreted his reports as indicating that, after some days, visual perception adapted and the world was seen upright again. Others claim that careful reading of his reports indicate that there

This research was supported by Grant \# GB-20511 from the National Science Foundation to Leon Festinger.

We wish to thank Julian Hochberg and Lloyd Kaufman for their helpful comments on the early draft of the article.

Requests for reprints should be sent to Leon Festinger, Psychology Department, Graduate Faculty, New School for Social Research, 65 Fifth Avenue, New York, New York 10003. was no visual perceptual change, but that only motor learning occurred. Some years later, Ewert (1930) reported a study, using several observers who wore a binocular inverting system for as many as 18 days. He reports that there was evidence of motor adjustment but absolutely no hint of any change in visual perception.

Research on the problem languished for many years but was powerfully revived by Kohler $(1951,1964)$. He reported remarkably complete instances of visual adaptation to inverting optical devices and to distortions produced by wearing spectacles containing wedge prisms. Subsequent studies have not reported such strong effects but have shown that adaptation to optically produced distortions such as displacement of the visual world (e.g., Hay \& Pick, 1966; see, also, a review by Kornheiser, 1976), tilt (e.g., Ebenholtz, 1973 ; Mikaelian $\&$ Held, 1964), and curvature does occur.

But the interpretation of these findings is still far from clear. Harris $(1963,1965)$ has argued convincingly that the adaptation involves not a change in visual perception, but rather a change in the felt position of 
limbs, head, and body. His argument is so persuasive that, if we are to pursue the question of change in visual perception, it seems wise to concentrate on situations for which the Harris explanation seems less cogent. One primary candidate for this would seem to be adaptation to optically produced curvature distortion (Hochberg, 1963). An observer, who wears spectacles with wedge prisms, bases mounted laterally, perceives straight vertical lines as curved, a perception that corresponds to the pattern of retinal stimulation. If, after a period of wearing these spectacles, such vertical lines appear straight again (or at least less curved than before), it seems plausible that true change in visual perception has occurred. The perception of the relationship among points on the retina itself has been altered; so it seems more difficult to explain this in terms of change in the felt positions of parts of the body. Let us, then, examine the data in this particular area.

\section{Is There Evidence of Visual Adaptation to Curvature Distortion?}

The answer to this question is yes. We will not attempt an exhaustive review of the literature but will mention only a few persuasive studies. Pick and Hay (1964) report on eight observers who wore prism spectacles oriented so that straight vertical contours were retinally curved. After wearing these spectacles for 42 days, these observers showed an average of $30 \%$ visual adaptation to the curvature distortion.

To evaluate properly these findings, one must remember that Gibson (1933) discovered that simple inspection of a curved line for a few minutes results in a similar effect. The magnitude of this "normalization" effect, however, is small and it cannot account entirely for the amount of perceptual adaptation Pick and Hay reported. Held and Rekosh (1963) report a study that conclusively eliminates the Gibson normalization effect as the sole explanation of such findings. Observers wore 20-diopter monocular prisms, bases mounted laterally, and walked around for one half hour in a darkened cylindrical room, the walls of which were covered with a random array of small luminous spots. Thus, there were no curved contours that could produce the Gibson effect. The relation between physical and retinal relative locations would, however, be the same as in the presence of actual contours. After one half hour, these observers showed $17 \%$ adaptation to curvature when asked to adjust a vertical line until it looked straight.

It must be noted that the effects reported above are small. A $30 \%$ adaptation, including the Gibson effect as a component, after 42 days of experience, is not very striking, but the effect is there. Perhaps the effects are small because, although movements of the head, body, or limbs while wearing such spectacles must conform to the relative physical locations if they are to be accurate, movement of the eyes must continue to be appropriate to the relative retinal locations.

Taylor (1962) pointed out that if the wedge prism were mounted on the eyeball, rather than on spectacles, then eye movements too would have to conform to the relative physical locations to be accurate. $\mathrm{He}$ had a contact lens containing an 11diopter prism fitted to his right eye and reports that, by simply scanning back and forth with his eye along a line, the total curvature distortion rapidly disappeared. These were rather informal observations and, in addition, the amount of curvature distortion produced by an 11-diopter prism with a curved front face would be very small indeed.

Festinger, Burnham, Ono, and Bamber (1967) repeated this study, fitting contact lenses containing 30-diopter prisms (bases down) to the right eyes of three observers. The only experience the observers had with this contact lens was to scan a horizontally oriented line, left eye occluded, with the head fixed in a biteboard. After 40 minutes of free eye movements scanning the line, there was an average of $44 \%$ adaptation when the line was physically straight (and therefore retinally curved) and $18 \%$ adaptation when the line was set so that the retinal image was straight (i.e., physically curved). Slotnick (1969), repeating this study with some additional conditions, re- 
ports very similar amounts of adaptation, namely, $36 \%$ and $16 \%$. It seems clear that one obtains some visual perceptual change with no experience other than eye movements when these movements, to be accurate, must conform to the physical relative locations rather than the discrepant retinal relative locations. It should be noted again, however, that the effects are rather small.

\section{Explanations of Change in Visual Perception}

The evidence indicates that there is some plasticity to the visual perceptual system and that some voluntary action or reaction to the environment, while wearing the optical distorting device, is necessary for perceptual change to occur. Thus, for example, in Held and Rekosh's (1963) previously mentioned study, if, instead of walking around the cylindrical room, the observer was passively wheeled around, no significant change in visual perception of curvature occurred. Another example can be cited from Slotnick's (1969) previously mentioned study. If the observer wearing the prism on a contact lens, instead of making free saccadic eye movements, followed a point moving slowly back and forth along the line, the results are quite different. In this latter condition, the eye engages in smooth pursuit eye movements and such movements, following a moving target, need only be oriented toward reducing small local errors on the retina. In this sense it resembles a passive movement condition. Here, the observers show no change in visual perception of curvature when the line is retinally straight and show a change that is quite consistent with the expected magnitude of the Gibson effect when the line is retinally curved.

Two kinds of theories have been proposed to account for such data. One of these, perhaps best exemplified by Held (1961), builds on the theoretical work of von Holst (1954). Because of the optical rearrangement, retinal information does not match the copy of the motor command. This mismatch leads gradually to a recoding of the retinal input resulting in motor learning and, presumably, in a change in the visual perception of curvature. A different kind of explanation was proposed by Taylor (1962) and somewhat elaborated by Festinger et al. (1967). For them the motor relearning is of primary importance. Because of the optical rearrangement, the efferent programs activated by the retinal input are in error. The voluntary activity with respect to the environment forces a change in the programs activated by the retinal input. Festinger et al. then propose not that the input is recoded but that visual perception is based on the efferent programs activated and held in readiness for use. Thus, the change in visual perception follows as a direct consequence of the motor learning.

From the existing data it is not possible to choose between these two different kinds of theory, nor is it possible to assess adequately the validity of either of them. Both require a close correspondence between motor relearning and change in visual perception, perhaps in a different temporal order. But, with respect to visual adaptation to curvature distortion, no one has ever adequately measured the course of motor relearning and compared it to the course of perceptual change. McLaughlin, Kelly, Anderson, and Wenz (1968) did attempt to answer a similar question. Observers fixated a light that was straight ahead and made a saccadic eye movement to another light that was $10^{\circ}$ in the periphery. During the saccade, the target light disappeared and was replaced by a light that was only $5^{\circ}$ away from the central fixation point. They found that during 11 such saccades there was a significant reduction in the magnitude of the saccade, that is, there was motor learning. They then asked their observers, while fixating the central light, to point (without sight of the hand) to the light $10^{\circ}$ away. They did not find a statistically significant change from before to after in the direction of pointing. One might argue, however, that 11 saccades was a rather small amount of experience.

The paradigm of the wedge prism on a contact lens, with eye movements being 
the only active experience permitted the observer, seems to be a feasible way to collect the relevant data. If, using such a paradigm, we can measure eye movements precisely and can also measure the course of change in visual perception, the existing theories could be more adequately evaluated. This was the purpose of the present study.

\section{Plan of the Study}

Instead of using an optical device, it is preferable to produce the desired rearrangement of the visual world by a computercontrolled visual display, the position of which is continuously contingent upon the eye position of the observer. Gourlay, Gyr, Walters, and Willey (1975) report a method for accomplishing this which is somewhat similar to the method we employ.

The advantages of such a procedure are (a) one is not limited to the very small curvature distortions that can be produced by a prism on a contact lens, (b) one can obtain highly accurate measures of eye position while allowing the observer to make reasonably large saccades, (c) one can produce any kind of contingency between eye position and display position, not only the single kind produced by a prism, and (d) one can study a single "distortion," namely, curvature, uncomplicated by displacement and other distortions introduced by prisms.

If an observer views a straight horizontal line through a wedge prism (base down), the optically transformed stimulus is curved (concave up). The lowest point on this curve is at that horizontal position determined by the perpendicular to the prism face. If the prism moves with the eye, as it does if mounted on a tightly fitted contact lens, then that minimum on the horizontal curve always coincides with the direction of gaze as the eye scans the curve. Thus, the eye, to be accurate, must move in a straight path even though the retinal image is curved. As we describe in detail later, this effect of a wedge prism mounted on a contact lens can be duplicated by displaying a curve that moves as the eye moves so that the minimum point always has a horizontal coordinate equal to that of the eye.

The above is not the only way of producing a situation in which accurate eye movements would have to be straight even though the retinal image is curved. We also describe in detail an arrangement in which the curve moves up and down as the eye moves so that the point on the curve that corresponds to the horizontal component of the direction of gaze is always at the identical vertical position. As we also explain later, one might expect that in this situation the learning of appropriate eye movements would be more difficult and might proceed more slowly. The theoretical expectation then would be that the rate of visual perception change would also be slower. In the experiment to be described, each of these two rearrangement types was used with each of three different magnitudes of curvature.

\section{Method}

\section{Observers}

Observers all had good uncorrected vision as measured by the Keystone Visual Survey Tests. Each served in only one condition for at least 5 successive days following calibration. (Some early observers were run 8 days but when it became apparent that no significant motor or perceptual changes occurred in the last few days, the experiment was shortened to 5 days.) Observers were naive with respect to the purpose of the experiment. All were volunteers and were paid for their time.

\section{Visual Display}

The observers viewed, in total darkness, a display consisting of three "parallel" curved lines, concave upward, separated vertically by $1^{\circ}$ and extending $22^{\circ}$ horizontally. Each line was composed of spots with a diameter of about 2.5 minutes of arc. The distance between the centers of adjacent spots was 3.3 minutes of arc. On the middle line were 5 small figures, each 9 minutes of arc on a side: (a) a square whose horizontal position was at the center of the display and straight ahead of the observer's right eye, (b) a circle $5^{\circ}$ horizontally to the right of center, (c) a diamond $5^{\circ}$ left of center, and (d) two " $\mathrm{X}$ "s, one $7^{\circ}$ left and one $7^{\circ}$ right of center. The curve of each of the three lines is given by $y=c x^{\frac{7}{3}}$, where $c$ determines the amount of curvature. Section A of Figure 1 shows what the display looked like. 
The visual displays were generated digitally by a Nova 2 computer linked, through a custom-designed oscilloscope control containing two 13-bit digital-toanalogue converters, to a Hewlett Packard 1310 display oscilloscope equipped with a P15 phospher and a contrast screen. The decay time of this phospher is less than $3 \mu \mathrm{sec}$ so that, when the display moved, it left no perceptible physical traces behind. The position of the display was adjusted for current eye position every 2 msec. Brightness was adjusted so that the display was clearly visible to the lightadapted observer, but not so high as to cause perceptible general illumination of the oscilloscope face. The observers viewed the display from a distance of $1 \mathrm{~m}$ with head held in place by a biteboard and forehead rest.

\section{Measurement of Eye Position}

The position of the observer's right eye (left eye always occluded) was monitored by a double Purkinje image eyetracker which has been described in detail elsewhere (Cornsweet \& Crane, 1973). Briefly, the eyetracker operates by measuring the relative position of the two images created by reflecting a beam of infrared light off the front surface of the cornea and the rear surface of the lens. The eyetracker's output consists of two continuous analog signals related to horizontal and vertical eye position over an approximately $16 \times 16^{\circ}$ field with a noise level less than 4 minutes of arc.

The accuracy of the eyetracker is not affected by translational movements of the head or eye, since these cause no relative motion of the two reflections. The tracker's output, however, is not linear with respect to direction of gaze; these nonlinearities vary somewhat from one observer to another. In addition, different observers required different scale factor adjustments, probably due to differences in the radius of curvature of the cornea, the rear of the lens, and the size of the eyeball. Further, the baseline varies somewhat from trial to trial with a given observer, depending on how he gets seated and into the biteboard-forehead rest. Hence, the accuracy of our eye position data is dependent on the accuracy of linearity, scale, and baseline corrections applied to it.

Accordingly, the first 2-hour session with each observer was devoted to gathering calibration data. The observer fixated a spot of light that jumped in a quasi-random path through 81 positions forming a $9 \times 9$ array, covering a $14^{\circ}$ square field. At each spot position, the median eye position was computed and recorded. The data from eight such trials were used to construct a two-dimensional matrix of correction vectors and to compute a scale factor for the observer.

During the experiment, a correction for baseline was computed at the start of each experimental trial. The voltage outputs of the eyetracker corresponding to the horizontal and vertical components of eye position were sampled every $2 \mathrm{msec}$ converted to digital form with 12 -bit resolution, corrected for linearity, scale, and baseline, and stored in the computer. Every $2 \mathrm{sec}$ the accumulated data were written out on magnetic tape for later analysis.

\section{Measurement of Perceived Curvature}

To obtain perceptual measures, the observer viewed the display shown in Section $A$ of Figure 1 with a bright spot added in the center of the square. By pressing a two-way switch up or down, the observer was asked, while fixating the center point, to adjust the curvature of the lines until they appeared straight. When satisfied with his setting, the observer pressed a second switch. The setting was then recorded and the curve repositioned for the next measurement. Four such measurements were taken in a row, two starting with the curves concave upward as shown in Figure 1 and two starting with the curves concave downward. As the curvature of the display lines changed during these measurements, the distance along the curve between adjacent spots composing the lines remained constant

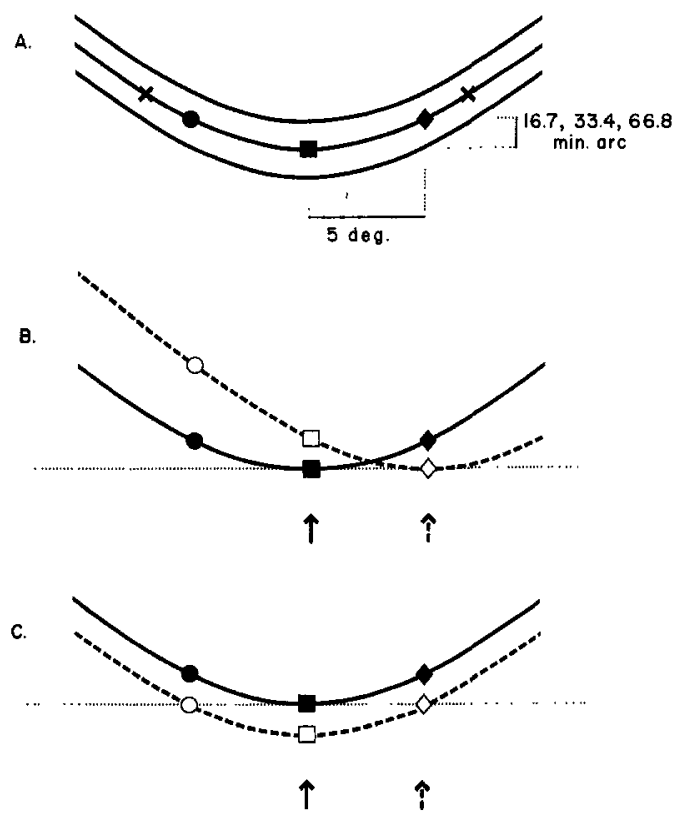

Figure 1. This is a diagram of the stimulus display. (A: Display when the eye is looking straight ahead showing its most important dimensions. $B$ and $C$ : Schematic diagrams of curve motion in the horizontal [B] and vertical [C] conditions. The solid curve indicates the display position when the eye is in the horizontal position given by the solid arrow. If the eye moves right, to the position of the dashed arrow, the display takes the position of the dashed curve. Whatever point on the curve is fixated has the same vertical location as indicated by the horizontal dotted line.) 
to avoid any extraneous cues. If, during the course of making these adjustments, the observer's direction of gaze was outside a $1^{\circ}$ square area surrounding the fixation point, the display disappeared leaving only the fixation point visible. The display reappeared when the eye returned to within the designated area. Thus, eye movements to scan the curves during the measurement were not possible.

\section{Design}

Each observer was assigned to one of the two eye movement contingency conditions. In both conditions, in order to fixate any point on the central curve of the display, the vertical component of the observer's direction of gaze would have to remain constant. This was accomplished as follows:

Horizontal curve movement (Condition $H$ ). In this condition, as the eye moved, the curves were shifted horizontally so that the minimum point on the curves was always at the horizontal coordinate of the direction of gaze. This is illustrated in Section B of Figure 1. The solid curve shows the position of the display when the observer's eye looked straight ahead. The dashed curve shows the position of the display when the observer's horizontal coordinate of gaze direction was $5^{\circ}$ to the right of the center. To simplify the illustration, only the center curve is shown. The lower and upper curves always remained parallel with this center curve. As shown in Figure 1 , the diamond, the square, and so on all retained their constant horizontal position. In Condition $\mathrm{H}$, one would expect that an observer, moving his eye from one point on the curve to fixate another point, would find himself fixating above the curve and would have to correct downward. If the observer learned to make different eye movements appropriate to the situation, this would involve a reduction in the vertical component of these eye movements.

Vertical curve movement (Condition V). In this condition, as the eye moved, the position of the curves shifted vertically so that whatever point on the curve the observer fixated would have the identical vertical position. This is illustrated in Section $C$ of Figure 1. Again the solid curve shows the position of the display when the horizontal component of the eye position was straight ahead. The dotted curve shows the position of the display if the observer moved his eye to fixate $5^{\circ}$ to the right of the center. The dashed horizontal line helps to show that the vertical position of the point to be fixated remains constant. In this condition, the expected errors of the eye movements, and the corrections necessary, are more complex than in Condition $\mathrm{H}$. When the observer moves his eye from the center to either the right or left, the direction of gaze would be above the curve and a downward correction would be necessary. When, however, he moves his eye toward the center, the direction of gaze would be below the curve and an upward correction would be needed. Thus, in Condition V, learning appropriate new eye movements should be more difficult.
Within Conditions $\mathrm{H}$ and $\mathrm{V}$, observers were assigned to one of three different magnitudes of curvature. A simple way to describe these curvature magnitudes, easily interpretable in terms of the task the observers were given, is to state the vertical distance of the circle and diamond above the square when the curve was in its central position. These three values were $16.7,33.4$, and 66.8 minutes of arc. Thus, the experiment consisted of six experimental conditions. Two observers were run in each condition.

\section{Course of the Experiment}

On the first day following calibration, each observer was given practice in adjusting the curves until they looked straight. We then ran two measurement trials (four adjustments each) to obtain a premeasure of perception. On subsequent days, each session started with one measurement trial, Following this measurement there were eight inspection periods (each 2 minutes long), another measurement trial, eight more inspection periods, and a final measurement trial.

For all inspection periods, the curves were displayed concave up. The observer was instructed to limit his eye movements to looking from the square to the circle, to the square, to the diamond, to the square, and so on. It was emphasized in the instructions that the observer was to look at the center of each of the figures. The upper and lower curves, and the $\mathrm{Xs}$ on the middle curve, were never to be fixated. They were included to provide added texture to the display.

The eye movements were restricted in this manner because, to compare Conditions $\mathrm{H}$ and $\mathrm{V}$, it is desirable to have the observers experience the same magnitude of error of eye movements. If free scanning were permitted, the two conditions would not have been comparable. In Condition $\mathrm{H}$, an eye movement from left to right, say, past the center point, would involve a larger vertical error than a movement to the center. In Condition V, however, an eye movement from the side, past center, to the other side, would involve a reduced vertical error. In the extreme, if a subject in Condition $\mathrm{V}$ moved his eye from, for example, $4^{\circ}$ left to $4^{\circ}$ right of center, no vertical error at all would be involved.

During the inspection periods the eyetracker occasionally lost the eye. This was usually caused by blinks or partial blinks since, as the eyelashes came down, the reflections from the eye would be degraded. It could take some seconds for the tracker to recapture the eye. When this happened, if nothing were done, unwanted movements of the display, unrelated to eye position, would have occurred. To eliminate this problem, anytime the observer blinked (signalled by an abnormal deviation in amount of reflected light) or the tracker lost the eye (signalled by deviant voltage outputs, since the tracker photocell slewed rapidly to an extreme position) the computer blanked the total display, replacing it with a flashing spot at center. The observers were in- 
structed, if this occurred, to fixate the flashing spot. As soon as the tracker recaptured the eye within one-half degree of the flashing spot, the display reappeared and scanning continued.

The observers were given rest periods between each 2-minute inspection period. It seemed desirable to prevent the observer from viewing contours during these rests, since it might undo learning that had occurred. On the other hand, it was desirable that the eye be light adapted at the beginning of each inspection period so that the very slight glow from the oscilloscope face would not be detectable. To achieve these objectives, the observer rested while wearing close fitting "ganzfeld" spectacles (made from ping pong balls; Hochberg, Triebel, \& Seaman, 1951) through which no contours could be seen.

\section{Analysis of Eye Movement Data}

The eye position records collected during inspection trials were analyzed by computer. Although the observers were instructed to make saccades that should have had a horizontal component of $5^{\circ}$, occasionally smaller and larger saccades were made. To simplify the analysis of the data, we limited ourselves to those saccades having a horizontal magnitude of between 4 and $6^{\circ}$. We refer to these as initial saccades. We also computed the magnitudes of corrective saccades. These were defined as having a horizontal component of less than $1^{\circ}$ and following an initial saccade in less than $500 \mathrm{msec}$ but more than $100 \mathrm{msec}$. If the intersaccadic interval was less than $100 \mathrm{msec}$, the two saccades were assumed to be a "preprogrammed" double saccade and were treated as one eye movement.

The data of main interest are the vertical magnitudes of both the initial and the corrective saccades. In Condition $\mathrm{H}$, for all initial saccades, eye movement error would be indicated by positive vertical components. In Condition $\mathrm{V}$, however, error would be reflected by a positive vertical component of saccades away from center and a negative vertical component of saccades toward center. Consequently, in Condition $\mathrm{V}$, in order to average the data, the vertical components of initial saccades toward center were inverted.

\section{Results and Discussion}

\section{Saccadic Eye Movements}

Do observers learn to make saccadic eye movements appropriate to the situation and, if they do, is the rate of learning slower in Condition $\mathrm{V}$ than in Condition $H$ ? Since the learning of appropriate eye movements involves only an adjustment of the vertical component of the saccade, we examined these vertical components for initial saccades and for corrective saccades.
These two measures show virtually identical results, both between conditions and over time. Consequently, we present the
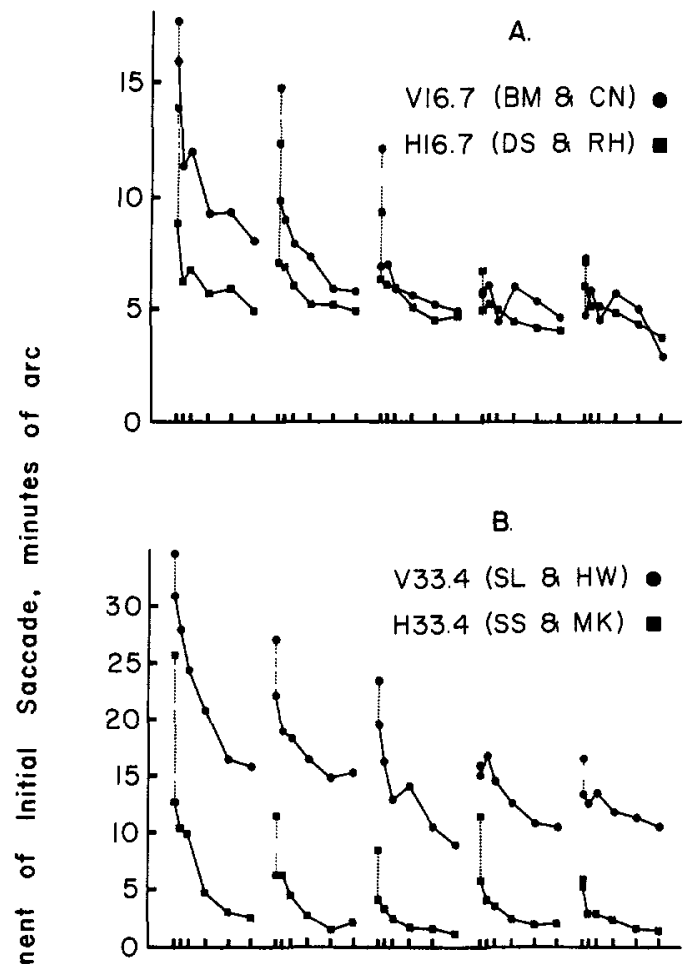

응

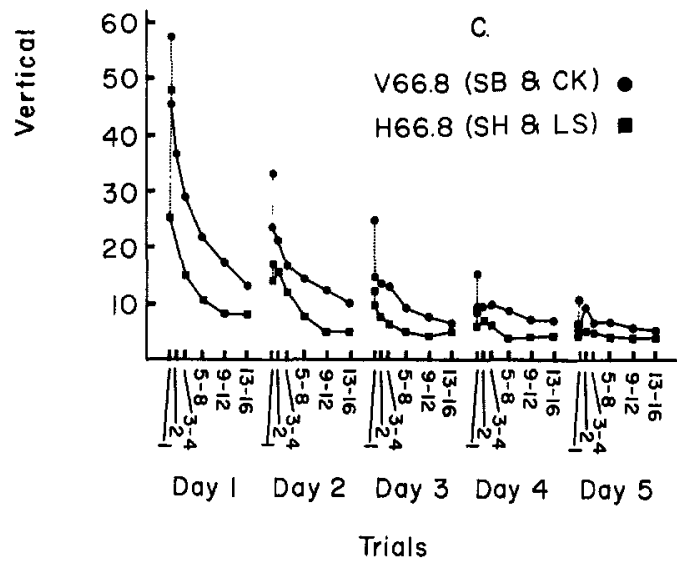

Figure 2. Mean vertical component of initial saccades in horizontal $(\mathrm{H})$ and vertical $(\mathrm{V})$ curve movement conditions for small (16.7 minutes arc; Section A), medium (33.4 minutes arc; Section B), and large (66.8 minutes arc; Section C) curvatures as a function of trial number over the 5 days of the experiment. (Subjects' initials appear in parentheses.) 
data only for the initial saccades. These data are shown in Figure 2. In this figure, each data point represents the mean vertical component of the initial saccades for two observers, each of the 5 successive days shown as a separate block of data points.

The first point in each day gives the mean vertical component of the first 10 saccades and is connected with a dotted line to the point indicating the mean for the first 2-minute trial (which includes the first 10 saccades). Each succeeding point gives the mean for the successive trials on that day, grouped as indicated on the abscissa. The unequal groupings of number of trials is for the purpose of showing clearly the course of change within each day. There were, on the average, 113 initial saccades per 2-minute trial.

Let us first look at how rapidly eye movements are relearned. It is clear from Figure 2 that the mean vertical component of the first 10 saccades on the first day is considerably higher than the mean for the first 2-minute trial. In other words, an appreciable amount of change in eye movements has taken place within the first 2 minutes of scanning the curve. This can be seen in more detail in Table 1 , which shows the average vertical component of the first 10 and the last 10 saccades in the first 2 -minute trial of the first day. It is clear that learning has taken place in each condition. It also appears that the relearning is

\section{Table 1}

Mean Vertical Component (minutes of arc) of First 10 and Last 10 Initial Saccades Made in the First Trial on the First Experimental Day

\begin{tabular}{ccc}
\hline Condition $^{\mathrm{a}}$ & First 10 & Last 10 \\
\hline H 16.7 & 13.87 & 8.58 \\
V 16.7 & 17.65 & 12.80 \\
H 33.4 & 25.82 & 13.17 \\
V 33.4 & 34.67 & 27.51 \\
H 66.8 & 47.86 & 14.57 \\
V 66.8 & 57.48 & 41.42 \\
\hline
\end{tabular}

${ }^{a} \mathrm{H}=$ horizontal curve motion; $\mathrm{V}=$ vertical curve motion; the number represents the curvature in minutes of arc, as explained in the text. faster in Condition $\mathrm{H}$ than in Condition $\mathrm{V}$. For each magnitude of curvature, the reduction in the vertical component is greater in Condition $\mathrm{H}$ than in Condition V. Indeed, there is evidence that, in Condition $\mathrm{H}$, some relearning has occurred during the first 10 saccades. In the three curvatures of Condition $\mathrm{V}$ the average vertical component of the first 10 saccades is rather close to the indicated magnitude of curvature but it is already less for the three $\mathrm{H}$ conditions.

Referring again to Figure 2, it can be seen that by the end of the first day, the vertical component has been greatly reduced, again more so for Condition $\mathrm{H}$ than for Condition V. A mean vertical component of zero would, of course, indicate complete adjustment of the initial saccades.

The course of learning over the 5 successive days is not surprising in view of the rapidity of learning within the first day. For all conditions there is a learning loss (increase in the vertical component) from 1 day to the next. This loss tends to become progressively less so that, by the fifth day, the mean for the first 10 saccades shows very little change from the end of the preceding day. Although the rate of learning is faster for Condition $\mathrm{H}$ than for Condition $\mathrm{V}$, by the end of the fifth day (except for the 33.4 curvature condition in which one Condition $\mathrm{V}$ observer shows aberrant data), the difference between Conditions $\mathrm{H}$ and $\mathrm{V}$ is negligible. With the same exception noted above, by the end of the last day the vertical component of the initial saccades is less than 5 minutes of arc. In other words, the eye movements have been almost completely adjusted to the experimental situation. Considering the normal visual experience that intervenes between the daily experimental sessions, the data clearly imply that the learning of the new eye movements has become conditional on the experimental situation.

One might argue that the observed diminution of the vertical component of the saccades might not reflect a true relearning of eye movements but might simply be due to conscious correction, that is, the realization on the part of the observers that they 
must move their eyes in a straight horizontal path. To assess this possibility, observers in the 16.7 and 66.8 curvature conditions were given an inspection period with the physical curvature equal to zero (straight lines) at the end of the experiment. If it were true that the observers had learned to move their eyes purely horizontally, regardless of the retinal location of the target, then the vertical components of the initial saccades scanning a straight line should be equal to zero. This is not the case. The average vertical component for the first 10 saccades scanning the straight line is $\mathbf{- 5 . 5}$ minutes for the four observers in the 16.7 curvature condition and -11.6 minutes for the four observers in the 66.8 curvature condition.

There are further reasons for doubting that conscious attempts to correct eye movements played a significant role. Such correction would require knowledge by the observer concerning the curve movements. Actually, in the 16.7 curvature Condition $\mathrm{H}$, the 16.7 curvature Condition $\mathrm{V}$, and the 33.4 curvature Condition V, observers did not perceive any clear movement of the curve. In the other conditions, movement was perceived. The similarity of the learning curves in all conditions in Figure 2 , however, argues against such perceived movement being a significant factor.

\section{Perceptual Adaptation}

We can now turn to an examination of the question of whether perceptual adaptation was in line with the relearning of appropriate eye movements. Figure 3 presents the relevant data. Each data point in this figure shows the average curvature of the display that looked straight to the observer, corrected for the constant error estimated from the eight measurements obtained prior to the first inspection trial on the first experimental day. For each day we have averaged the measurements made after 8 inspection trials and after all 16 inspection trials. Each data point thus represents the mean of eight measurements for each of two observers. To the right of each section of Figure 3 is a vertical line at the ends of which are horizontal bars that represent the mean across all 5 days for each of the two observers. Complete perceptual adaptation would be indicated by measurements of $16.7,33.4$, and $66.8 \mathrm{~min}$ utes of arc for the respective curvature conditions. That is, if perceptual adaptation were complete, the inspection curve would have appeared to be straight to the observer.

It is clear that perceptual adaptation is small and does not at all resemble the changes in the saccadic eye movements, either in magnitude or in time course. The largest absolute amount of perceptual adaptation occurs in the 33.4 curvature Condition $\mathrm{H}$ (Section $\mathrm{B}$ of Figure 3), but even there it is small, averaging about 5 minutes of arc. This is very different from the eye movement adjustment of about 30 minutes of arc for this condition (Section B of Figure 2). The largest percentage change in perception of curvature occurs in the 16.7 curvature Condition $\mathrm{H}$ (Section $\mathrm{A}$ of Figure 3) but even here a perceptual change of 4 minutes of arc is not commensurate with the change in eye movements (Section A of Figure 2). The difference between the amount of eye movement change and the amount of perceptual adaptation is most vividly seen by comparing Figure 3's Section C with Figure 2's. In the 66.8 curvature conditions, the eye movement change was more than 60 minutes of arc. The perceptual change, however, amounts to about 2 minutes of arc for Condition $\mathrm{H}$ and is essentially zero for Condition $V$. The temptation to conclude that perceptual adaptation has nothing to do with relearning the eye movements is strengthened if we look at the time course of adaptation over days. While the eye movement relearning tended to increase progressively from day to day, there is no such tendency whatsoever for the measures of perceptual adaptation.

There are some aspects of the data, however, that must be dealt with before accepting a conclusion of total independence between eye movements and perceptual adaptation. In Condition $\mathrm{H}$, in which eye movement relearning was faster, there is 
A.

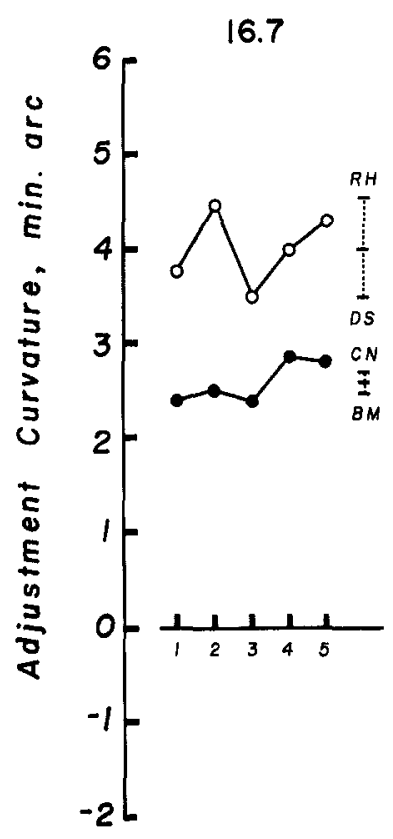

B.

33.4

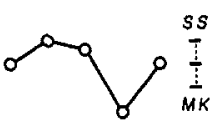

$\mathrm{HO}$

$\checkmark$

\section{8}



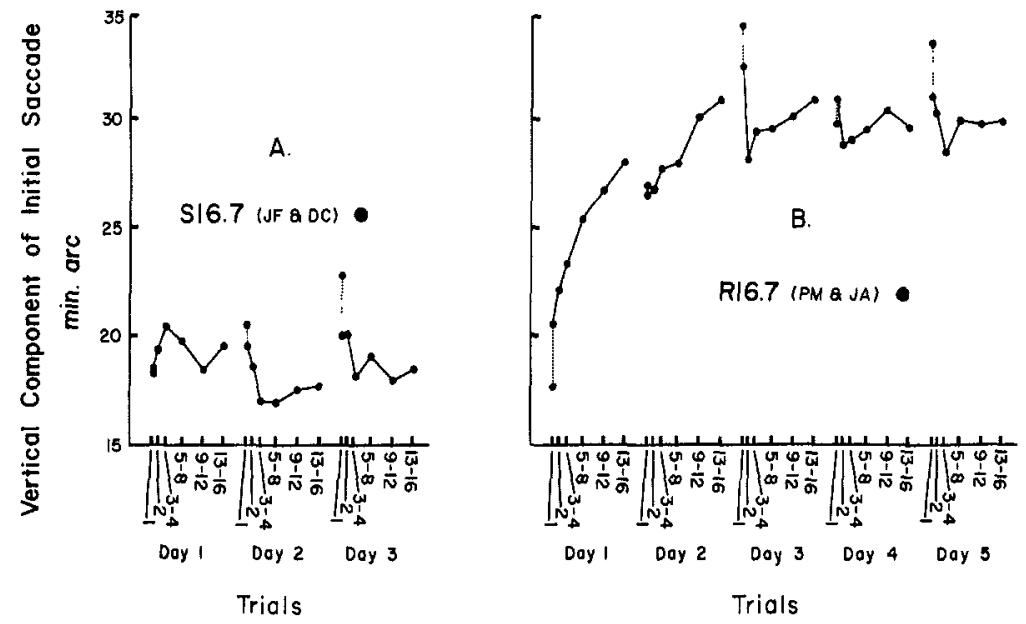

Figure 4. Mean vertical component of initial saccades in S (stationary; Section A) and R (reverse; Section B) curve movement conditions. (Display curvature is 16.7 minutes arc. Subjects' initials appear in parentheses.)

to be appropriate to a retinal image of a curve of twice the magnitude of the display curve, then any perceptual adaptation that was related to the reprogramming of eye movements would be in the opposite direction from Conditions $\mathrm{H}$ and $\mathrm{V}$. It is not possible to arrange this reversal paralleling the simple relearning requirements of Condition $\mathrm{H}$, since the display would quickly be driven vertically off the scope face. One can, however, arrange this reversal paralleling the somewhat more complex relearning requirements of Condition $V$. Thus, in this reverse (R) condition, the display moved $u p$ as the eye moved to either of the side figures and moved down as the eye moved toward the center figure. If the eye movements were reprogrammed to double the vertical component of the initial saccade, and if perceptual adaptation was related to this relearning of eye movements, then a concave-down curve should appear straight to the observers. Or if the normalization effect which may be omnipresent counteracts this, at least the perceptual change should differ significantly from that in Condition V. To assess this, two observers were run in Condition $R$, with a curvature of 16.7 , for 5 consecutive days. Except for the reversal of the contingency, all aspects of the ex- perimental procedure were exactly as already described.

\section{Additional Results}

Figure 4 presents the eye movement data (vertical component of the initial saccades) for Conditions $\mathrm{S}$ and $\mathrm{R}$. Not surprisingly, in Condition $\mathrm{S}$ there is little if any change in the vertical component of the saccades. In Condition $\mathrm{R}$ we observe the same kind of relearning of eye movements (in the opposite direction of course) as in Condition $\mathrm{V}$. The average vertical component of the first 10 saccades on the first experimental day is approximately appropriate to the curvature of 16.7 minutes of arc. There is rapid learning; the magnitude of the vertical component on the fourth and fifth days hovers around 30 minutes of arc, about 5 minutes short of complete relearning. This is very similar, in reverse, to the data for the 16.7 curvature Condition $\mathrm{V}$ shown in Section A of Figure 2.

Let us now turn our attention to the data on perception which are presented in Figure 5. Here there is no hint of any relationship between eye movement relearning and perceptual adaptation. The perceptual measurements in Condition S are very similar to the comparable data in the 16.7 curvature Condition V (Section A of Figure 
A.

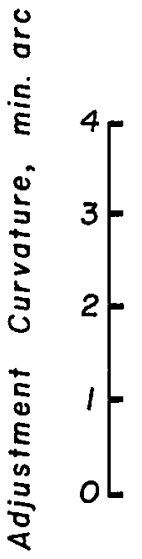

516.7

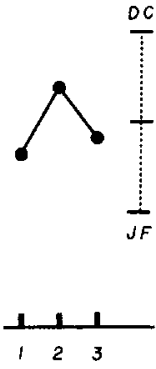

Day
B.

RI6.7
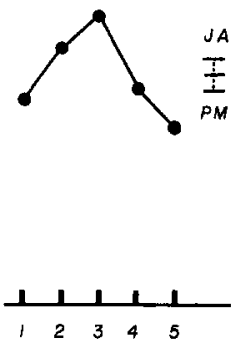

Day

Figure 5. Mean corrected settings of the adjustable curve in S (stationary; Section A) and R (reverse; Section B) conditions. (DC, JF, JA, and PM are subjects' initials.)

3). More striking, and more persuasive, is the fact that the perceptual measures in Condition $\mathrm{R}$ are indistinguishable from those in the 16.7 curvature Condition $\mathrm{V}$. We obtain nearly identical perceptual measurements in two experimental conditions in which almost complete eye movement relearning has taken place in opposite directions.

\section{Conclusions}

The data we have presented force the conclusion that, at least in this experimental situation, there is no relationship between the relearning of eye movements and perceptual change. In Condition $\mathrm{V}$, in spite of nearly complete eye movement adjustment, there is no evidence of any perceptual change whatsoever, excluding the Gibson normalization effect. In Condition $\mathrm{H}$, however, perceptual change did occur but was clearly unrelated to the relearning of eye movements.

We have shown that the perceptual change in Condition $\mathrm{H}$ is significantly greater than in Condition V. The implication is, of course, that perceptual change in Condition $\mathrm{H}$ is greater than can be attributed to the normalization effect. To provide

some additional direct evidence concerning this, we measured the normalization effect, for 1 day only, for two subjects scanning the 16.7 curve and for two scanning the 33.4 curve. The results from the first day of the two previously mentioned 16.7 curvature Condition $\mathrm{S}$ subjects were combined with these data. The mean adjustments were 2.64 and 2.34 minutes of arc for the 16.7 and 33.4 curves, respectively. The comparable values for the first day of the subjects in the 16.7 and 33.4 curvature Condition $\mathrm{H}$ were 3.78 and 5.24. The perceptual adaptation in Condition $\mathrm{H}$ is significantly greater than in Condition $\mathrm{S}$, $F(1,6)=6.82, p<.05$.

To assess the generality of our results, it is desirable to compare them with the results of other studies on adaptation to curvature distortion. It should be pointed out that previous studies, having used wedge prisms, are all similar to our Condition $\mathrm{H}$. All of these studies found perceptual adaptation to curvature distortion and, in this condition, we find such adaptation also. The question arises as to whether the amount of adaptation in this study resembles the amount obtained in previous studies or whether our results are smaller in magnitude, perhaps indicating that our restricted situation somehow prevented perceptual adaptation.

It is not possible, in all of the studies reported in the literature, to calculate accurately the amount of curvature produced by the prisms. These curvatures, however, are definitely not very large; there is probably no study in which the retinal curvature was greater than the smallest curvature used in the present experiment, namely, a deviation of 16.8 minutes of arc at a distance of $5^{\circ}$ from the center. Festinger et al.'s (1967) and Slotnick's (1969) contact lens studies produced curvatures of only between 5 and 10 minutes of arc (measures we obtained on the same curvanometer used in those studies). Thus, reports of $30 \%$ or $40 \%$ adaptation represent perceptual change of only a few minutes of arc, quite comparable to the perceptual changes that we found. There seems no reason to believe that anything in our ex- 
perimental situation hindered perceptual adaptation.

If the perceptual adaptation in Condition $\mathrm{H}$ is not, as seems clear, related to the relearning of appropriate eye movements, what are the conditions that produce it? The answer to this is by no means clear, but we can make one suggestion. One may think of Condition $\mathrm{H}$ as producing a situation in which the curve is stabilized on the retina with respect to the horizontal component of an eye movement. Vertical eye movements, of course, change the retinal locations of the curve but a purely horizontal eye movement, if the curve extended across the entire visual field, would not produce any change in the points stimulated on the retina. This is a situation which, in a normal visual environment, would only be produced by a straight line. Thus, it is possible that the perceptual change obtained in Condition $\mathrm{H}$ is due to this property of the situation. This is of course not the case in Condition $\mathrm{V}$ in which we found no perceptual change.

If our results are correct, and generalizable, they refute most of the theoretical attempts to explain perceptual adaptation to curvature distortion. Theories such as those offered by Held (1961), Taylor (1962), and Festinger et al. (1967) clearly require a close relationship between motor relearning and perceptual change, regardless of assumptions of the direction of causality between the two. Harris' (1965) theory is, of course, unaffected by our results. He emphasizes changes in felt position of parts of the body and implies that perception of relative retinal location is relatively unalterable. It may be that he is correct.

It is important, however, to point out the difficulties in generalizing our results in any sweeping manner. Our experimental situation was very restrictive and differs in possibly important aspects from the situations employed by other studies. Technical limitations forced us to restrict the field of view to a small fraction of what is normal. Technical limitations also forced us to use a visual display that was relatively textureless. The observers saw only three curves, with the markers on the central curve, in an otherwise totally dark and totally contourless field. We do not know what effects these aspects of the situation may have had, but they do warrant some caution about our general conclusions.

Perhaps of even greater significance is that, by instruction, the observers' experience was primarily limited to making saccades with about a $5^{\circ}$ horizontal component. The a priori reasons for doing this, to enable clear comparison between Conditions $\mathrm{H}$ and $\mathrm{V}$, seemed compelling but, post hoc, may have been unfortunate in view of the results. It is possible that if the eye movements had been reprogrammed for a wide range of magnitudes of saccades, different results would have been obtained.

\section{References}

Cornsweet, T. N., \& Crane, H. D. Accurate twodimensional eye tracker using first and fourth Purkinje images. Journal of Optical Society of America, 1973, 63, 921-928.

Ebenholtz, S. H. Optimal input rates for tilt adaptation. American Journal of Psychology, 1973, 86, 193-200.

Ewert, P. H. A study of the effect of inverted retinal stimulation upon spacially coordinated behavior. Genetic Psychology Monographs, 1930, 7, 177-363.

Festinger, L., Burnham, C. A., Ono, H., \& Bamber, D. Efference and the conscious experience of perception. Journal of Experimental Psychology Monograph, 1967, 74(4, Whole No. 637).

Gibson, J. J. Adaptation, after-effect and contrast in perception of curved lines. Journal of Experimental Psychology, 1933, 16, 1-31.

Gourlay, K., Gyr, J. W., Walters, S., \& Willey, R. Instrumentation designed to simulate the effects of prisms used in studies of visual rearrangement. Behavior Research Methods and Instrumentation, 1975, 7, 294-300.

Harris, C. S. Adaptation to displaced vision : Visual, motor or proprioceptive change? Science, 1963, $140,812-813$

Harris, C. S. Perceptual adaptation to inverted, reversed and displaced vision. Psychological Review, 1965, 72, 419-444.

Hay, J., \& Pick, H. Visual and proprioceptive adaptation to optical displacement of the visual stimulus. Journal of Experimental Psychology, 1966, 71, 150-158.

Held, R. Exposure history as a factor in maintaining stability of perception and coordination. Journal of Nervous and Mental Diseases, 1961, 132, 26-32.

Held, R., \& Rekosh, J. Motor-sensory feedback and the geometry of visual space. Science, 1963, 141, $722-723$. 
Hochberg, J. On the importance of movementproduced stimulation in prism-induced aftereffects. Perceptual Motor Skills, 1963, 16, 544.

Hochberg, J. E., Triebel, W., \& Seaman, G. Color adaptation under conditions of homogeneous visual stimulation (Ganzfeld). Journal of Experimental Psychology, 1951, 41, 153-159.

Kohler, I. Über aufbau und wandlungen der wahrnehmungswelt. Österreichische Akademie der Wissenschaften, Sitzungsberichte, Philosophischhistorische Klasse, 1951, 227, 1-118.

Kohler, I. [The formation and transformation of the perceptual world] (trans, by H. Fiss). Psychological Issues, 1964, 3(4), 1-173.

Kornheiser, A. S. Adaptation to laterally displaced vision: A review. Psychological Bulletin, 1976, 83, 783-816.

McLaughlin, S. C., Kelly, M. J., Anderson, R. E., \& Wenz, T. G. Localization of a peripheral target during parametric adjustment of saccadic eye movements. Perception \& Psychophysics, 1968, 4, 45-48.
Mikaelian, H., \& Held, R. Two types of adaptation to an optically rotated visual field. American Journal of Psychology, 1964, 77, 257-263.

Pick, H. L., \& Hay, J. C. Adaptation to prismatic distortion. Psychonomic Science, 1964, 1, 199-200.

Slotnick, R. S. Adaptation to curvature distortion. Journal of Experimental Psychology, 1969, 81, $441-448$.

Stratton, G. M. Some preliminary experiments on vision without inversion of the retinal image. Psychological Review, 1896, 3, 611-617.

Stratton, G. M. Upright vision and the retinal image. Psychological Review, 1897, 4, 182-187.

Taylor, J. G. The behavioral basis of perception. New Haven, Conn.: Yale University Press, 1962.

von Holst, E. Relations between the central nervous system and the peripheral organs. British Journal of Animal Behavior, 1954, 2, 89-94.

Received September 27, 1976 